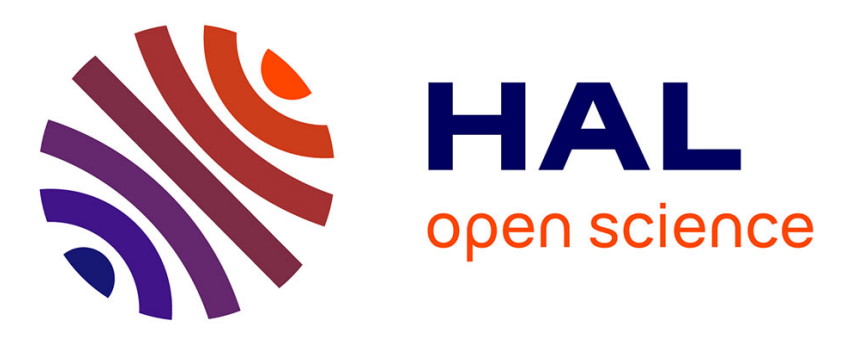

\title{
Feedback Control with Equilibrium Revision for CMG-Actuated Inverted Pendulum
}

Igor Ryadchikov, Semyon Sechenev, Nikita Mikhalkov, Andrey Biryuk, Alexander Svidlov, Aleksandr Gusev, Dmitry Sokolov, Evgeny Nikulchev

\section{To cite this version:}

Igor Ryadchikov, Semyon Sechenev, Nikita Mikhalkov, Andrey Biryuk, Alexander Svidlov, et al.. Feedback Control with Equilibrium Revision for CMG-Actuated Inverted Pendulum. Proceedings of 14th International Conference on Electromechanics and Robotics "Zavalishin's Readings", pp.431-440, 2020, 10.1007/978-981-13-9267-2_35. hal-02282908

\section{HAL Id: hal-02282908 https://hal.inria.fr/hal-02282908}

Submitted on 10 Sep 2019

HAL is a multi-disciplinary open access archive for the deposit and dissemination of scientific research documents, whether they are published or not. The documents may come from teaching and research institutions in France or abroad, or from public or private research centers.
L'archive ouverte pluridisciplinaire HAL, est destinée au dépôt et à la diffusion de documents scientifiques de niveau recherche, publiés ou non, émanant des établissements d'enseignement et de recherche français ou étrangers, des laboratoires publics ou privés. 


\title{
Feedback control with equilibrium revision for CMG- actuated inverted pendulum
}

\author{
Igor Ryadchikov ${ }^{1}$, Semyon Sechenev ${ }^{1}$, Nikita Mikhalkov ${ }^{1}$, Andrey Biryuk ${ }^{1}$, \\ Alexander Svidlov ${ }^{1}$, Aleksandr Gusev ${ }^{1}$, Dmitry Sokolov ${ }^{2}$ and Evgeny Nikulchev ${ }^{3}$ \\ ${ }^{1}$ Kuban State University, 149 Stavropolskaya Street, 350040, Krasnodar, Russia \\ alexandrgsvegmail.com \\ ${ }^{2}$ UMR 7503, Université de Lorraine, CNRS, Vandœuvre-lès-Nancy, France \\ ${ }^{3}$ MIREA - Russian Technological University, 78 Vernadsky Avenue, Moscow 119454, Russia
}

\begin{abstract}
The article centers round the problem of design a feedback control for the control moment gyroscope (CMG) -actuated inverted pendulum with online equilibrium revision after the center of mass displacement. The methodology for the control synthesis is the Linear Quadratic Regulator. The equations of motion for the model of the inverted pendulum are derived. The control is synthetized and implemented both in an experimental plant and in a simulation model. The results of the experiment and simulation show the reliability of the synthetized control, which is proved to be able to deal with the center of mass displacement.
\end{abstract}

Keywords: CMG, inverted pendulum, feedback control, equilibrium revision.

\section{Introduction}

The dynamic stabilization of the walking robot is a growing direction of research [1], as such robots are created for use in the human environment, where the use of wheeled vehicles is difficult or impossible [2, 3]. The major difficulty of designing walking humanoid robots is providing compensation for deviations of the robot body from the equilibrium position arising when the machine is walking [4]. In our study, we consider a nonatropomorphic stabilization system for a walking robot based on the use of the compensating moment of a control moment gyroscope (CMG) to return the inverted pendulum which models the robot's body to the equilibrium position. The inverted pendulum is one of the common and adequate mechanical models to study the problem of the dynamic stabilization of a walking robot [5]. The CMG for inverted pendulum balancing has been intensively studied in recent years. In work [6] there was suggested a global change of coordinates to transform the dynamics of the system into a lower nonlinear subsystem and stabilize the system with a control Lyapunov function. Another research [7] was devoted to creation of a model predictive controller (MPC) to control such a system. A wearable scissored-pair control moment gyroscope for human balance assist was discussed in [8]. Nevertheless, it should be noted that in the transition from mathematical models to the creation of real walking machines, the problem of the error in determining the center of mass (CoM) of the robot remains unsolved. To solve this problem, it is possible to 
design state observers, as in [9]. The state observers provide estimates of the internal state of the real system to compensate the sensor error. However, under certain conditions it is possible to treat the CoM displacement as the sensor error.

In this article, we instead demonstrate the synthesis of the LQR (Linear-quadratic regulator) control that is reliable enough in the case of equilibrium revision due to the CoM displacement. The LQR is usually implemented when the system dynamics is described by a set of linear differential equations and the cost to be minimized (usually a sum of key deviations from the desired state of the system) is described by a quadratic function. In Section 2, the equations of motion for the considered model of an inverted pendulum with a CMG are presented and the general form of the desired control is derived. Section 3 presents the parameters of an experimental plant and a simulation model with a regulator that implements the synthetized control. Section 4 compares the experimental data taken from the plant and obtained in MATLAB / Simulink when modeling the additional load leading to the equilibrium revision. Section 5 concludes the article.

\section{Equations and mathematics}

The structure considered in this paper (see Fig. 1) consists of three parts: the CMG, its binding and the inverted pendulum.

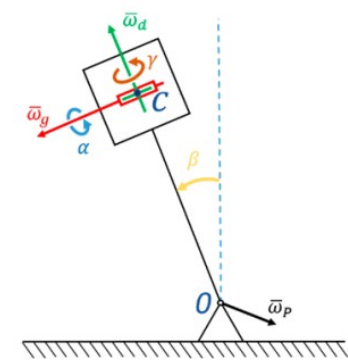

Fig. 1. The robot mechanical model.

The position of the structure in space is uniquely defined by three angles: $\alpha$ which is the angle of rotation of the binding relative to the inverted pendulum, with $\alpha=0$ corresponding to the position of the axis of rotation of the CMG along the way of the axis of rotation of the inverted pendulum; $\beta$ is the angle of rotation of the axis of the inverted pendulum relative to the vertical; $\gamma$ is the angle of rotation of the CMG relative to its binding.

It is clear that $\gamma$ is a cyclic variable. The angular velocities are denoted as follows: $\bar{\omega}_{p}$ is the angular velocity of the inverted pendulum relative to the fixed 
frame; $\bar{\omega}_{g}$ is the angular velocity of the binding relative to the inverted pendulum; $\bar{\omega}_{d}$ is the angular velocity of the CMG relative to its binding.

The following equalities hold:

$\omega_{p}=\dot{\beta}, \omega_{g}=\dot{\alpha}, \omega_{d}=\dot{\gamma}$.

We assume that centers of mass of the CMG and its binding coincide (point $C$ in Fig. 1) and they are located at the intersection of the axis of rotation of the binding, the axis of rotation of the CMG and the axis of the inverted pendulum. In the subsections 2.1 and 2.2 we derive the equations of motion and synthesize a control based on the assumption that the CoM of the inverted pendulum lies on its axis. In the subsection 2.3. we show that the synthesized control is applicable also for the case of the displaced CoM. In the case when the position of the CoM is unknown, the synthesized control can be used to automatically search for the equilibrium position.

\subsection{Equations of motion}

Let the CoM of the inverted pendulum lie on its axis. Then the total potential energy is:

$$
\Pi=\Pi_{0} \cos \beta,
$$

where $\Pi_{0}$ is the potential energy of the whole structure when $\beta=0$.

The full kinetic energy of the system can be written as follows:

$$
T=\frac{1}{2}\left(J_{p} \dot{\beta}^{2}+\left(m_{g}+m_{d}\right) h^{2} \dot{\beta}^{2}+I_{d}(\dot{\gamma}-\dot{\beta} \sin \alpha)^{2}+\left(J_{d}+J_{g}\right)\left(\dot{\alpha}^{2}+\dot{\beta}^{2} \cos ^{2} \alpha\right)+I_{g} \dot{\beta}^{2} \sin ^{2} \alpha\right),
$$

where $h=O C, m_{d}$ is the mass of the CMG, $m_{g}$ is the mass of the binding, $J_{p}$ is the moment of inertia of the inverted pendulum relative to the axis of rotation passing through the point O. $I_{d}$ and $I_{g}$ are the moments of inertia of the CMG and its binding relative to the CMG rotation axis, $J_{d}$ and $J_{g}$ are the moments of inertia of the CMG and its binding with respect to any perpendicular axis passing through the point $C$. It is assumed that the moments of inertia are independent with respect to the choice of this axis.

The Lagrangian of the structure is given by $L=T-\Pi$. The equations of motions have the form:

$$
\begin{aligned}
& \frac{d}{d t} \frac{\partial L}{\partial \dot{\alpha}}-\frac{\partial L}{\partial \alpha}=\tau_{a}, \\
& \frac{d}{d t} \frac{\partial L}{\partial \dot{\beta}}-\frac{\partial L}{\partial \beta}=0, \\
& \frac{d}{d t} \frac{\partial L}{\partial \dot{\gamma}}-\frac{\partial L}{\partial \gamma}=\tau_{d} .
\end{aligned}
$$

where $\tau_{a}$ is the actuator torque, $\tau_{d}$ is the CMG motor torque. The actuator rotates the binding with respect to the inverted pendulum, while the CMG motor rotates the CMG relatively to its binding. With elementary transformations the equations of motion get the form: 


$$
\begin{aligned}
& \left(J_{d}+J_{g}\right) \ddot{\alpha}+I_{d} \dot{\gamma} \dot{\beta} \cos \alpha+\left(J_{d}+J_{g}-I_{d}-I_{g}\right) \dot{\beta}^{2} \cos \alpha \sin \alpha=\tau_{a} \text {, } \\
& \ddot{\beta} \dot{\zeta} \quad \text { (2) }
\end{aligned}
$$

$$
I_{d}(\ddot{\gamma}-\dot{\beta} \dot{\alpha} \cos \alpha-\ddot{\beta} \sin \alpha)=\tau_{d} .
$$

\subsection{Control design}

Further we assume that the CMG motor turnover controller is supporting ideally the given angular velocity of the CMG $\dot{\gamma} \equiv \Gamma$, in other words the turnover controller chooses $\tau_{d}=I_{d}(\dot{\beta} \dot{\alpha} \cos \alpha+\ddot{\beta} \sin \alpha)$ and the equation (3) holds automatically.

Since most electric motors applied in practice do not allow controlling the torque, instead they are controlled by setting the speed of rotation of the shaft, it seems reasonable to assume that $\tau_{a}$ is such that the equation (1) holds automatically and we can control the velocity $\dot{\alpha}$ directly. These assumptions are quite natural and not restrictive as the quantities $\dot{\alpha}, \dot{\beta}, \ddot{\alpha}$ и $\ddot{\beta}$ in practice will be respectively not so high and the actuator is powerful enough to provide practically instant transition to any given velocity $\dot{\alpha}$.

So, the equations (3) and (1) hold due to assumptions above. It remains to analyze equation (2). The task is to drive $\beta$ to zero controlling the velocity $\dot{\alpha}$. The additional task is to lead $\alpha$ to zero as well.

By linearizing the equation (2) near the equilibrium position $\beta=0, \alpha=0, \dot{\beta}=0$, $\dot{\alpha}=0$ we get

$$
B_{0} \ddot{\beta}-\Pi_{0} \beta=I_{d} \Gamma \dot{\alpha}
$$

where $B_{0}=J_{p}+\left(m_{g}+m_{d}\right) h^{2}+J_{d}+J_{g}$ is the moment of inertia of the whole structure relative to the axis of rotation passing through the fulcrum $\mathrm{O}$ provided that $\beta=\alpha=0$. Having brought the equation to the normal form we get the following:

$$
\ddot{\beta}=M \beta+F \dot{\alpha},
$$

where $M=\frac{\Pi_{0}}{B_{0}}, \quad F=\frac{I_{d} \Gamma}{B_{0}}$.

Next, we proceed to the control design. Let $u=\dot{\alpha}$ be the control. Introduce the notation $a(t)=\int_{0}^{t} \alpha(s) d s$. Then the control problem may be written as:

$$
\left\{\begin{array}{c}
\ddot{\beta}=M \beta+F u, \\
\ddot{a}=u .
\end{array}\right.
$$

Equivalently, in the matrix form: 


$$
\frac{d}{d t}\left(\begin{array}{c}
\dot{\beta} \\
\beta \\
\dot{a} \\
a
\end{array}\right)=\left(\begin{array}{cccc}
0 & M & 0 & 0 \\
1 & 0 & 0 & 0 \\
0 & 0 & 0 & 0 \\
0 & 0 & 1 & 0
\end{array}\right)\left(\begin{array}{c}
\dot{\beta} \\
\beta \\
\dot{a} \\
a
\end{array}\right)+\left(\begin{array}{c}
F \\
0 \\
1 \\
0
\end{array}\right) u .
$$

The aim of the control is to make the point $\beta=\dot{\beta}=a=\dot{a}=0$ of the phase space of the system (4) asymptotically stable.

The control is sought in the form of a linear state feedback:

$$
u=-\left(\begin{array}{llll}
k_{\dot{\beta}} & k_{\beta} & k_{\dot{a}} & k_{a}
\end{array}\right)\left(\begin{array}{c}
\dot{\beta} \\
\beta \\
\dot{a} \\
a
\end{array}\right),
$$

where $k_{\beta} \quad k_{\beta} \quad k_{\dot{a}} \quad k_{a}$ are the control parameters.

Introducing

$$
x=\left(\begin{array}{c}
\dot{\beta} \\
\beta \\
\dot{a} \\
a
\end{array}\right), A=\left(\begin{array}{cccc}
0 & M & 0 & 0 \\
1 & 0 & 0 & 0 \\
0 & 0 & 0 & 0 \\
0 & 0 & 1 & 0
\end{array}\right), b=\left(\begin{array}{c}
F \\
0 \\
1 \\
0
\end{array}\right),
$$

the matrix form of the equation (4) may be written as following:

$$
\frac{d}{d t} x=A x+b u \text {. }
$$

The problem of building a linear control for (5) has the solution as $\operatorname{det}\left(b, A b, A^{2} b, A^{3} b\right) \neq 0[10 \S 2,3]$.

The control parameters $k_{\dot{\beta}} \quad k_{\beta} \quad k_{\dot{a}} \quad k_{a}$ can be found using the LQR technique [11]. Numerical simulation shows a sufficient robustness of the proposed technique, i.e. when the control parameters are "frozen", the system remains stabilized even after a significant change in the masses of the system components.

\subsection{The impact of CoM displacement}

Suppose that as a result of some additional mechanical load, the CoM of the system is not anymore on the axis OC. At the same time, we do not know in advance the new position of the CoM. Given the robustness of the LQR method, this is mathematically expressed through the presence of some unknown constant error in determining the angle $\beta$. In other words, instead of the true direction to the vertical $\beta$ the sensors give $\beta+\Delta$. This in turn leads to a shift in the control and the control takes the form:

$$
u=-\left(\begin{array}{llll}
k_{\beta} & k_{\beta} & k_{\dot{a}} & k_{a}
\end{array}\right)\left(\begin{array}{c}
\dot{\beta} \\
\beta \\
\dot{a} \\
a
\end{array}\right)-k_{\beta} \Delta,
$$


the system (5) with this modified control also has the asymptotically stable stationary solution that can be found by solving the following system of linear equations:

$$
\begin{gathered}
(A-b k) x=b k_{\beta} \Delta . \\
\left(\begin{array}{c}
-F k_{\dot{\beta}} \dot{\beta}-\left(F k_{\beta}-M\right) \beta-F k_{\dot{a}} \dot{a}-F k_{a} a=F k_{\beta} \Delta, \\
\dot{\beta}=0, \\
-k_{\dot{\beta}} \dot{\beta}-k_{\beta} \beta-k_{\dot{a}} \dot{a}-k_{a} a=k_{\beta} \Delta, \\
\dot{a}=0 .
\end{array}\right.
\end{gathered}
$$

Having solved the system above we find that

$$
\dot{\beta}=\dot{a}=\beta=0, a=\frac{-k_{\beta}}{k_{a}} \Delta .
$$

It follows from the obtained equalities that even with a shift in the CoM the system considered will stabilize in a position where the CoM is located above the fulcrum. In this case, the axis of rotation of the CMG will be in the plane of rotation of the inverted pendulum.

\section{Implementation of the controller}

To implement the controller, we designed a MATLAB/Simulink model of the inverted pendulum with the CMG using the Simscape Multibody simulation library and assembled an experimental plant.

The mechanical structure of the CMG of the experimental plant is given in Table 1.

Table 1. The plant CMG mechanical structure

\begin{tabular}{cc}
\hline Element & Mass $(\mathrm{kg})$ \\
\hline the power gyro (with the protective hull & \\
for the flywheel, the engine, the servo motor & 5.3 \\
actuators, the bearing units) & 1.1 \\
the control gyro & 2.5 \\
the common frame & 0.6 \\
the pendulum mount & 1.0 \\
the pendulum hinge &
\end{tabular}

The flywheel inside the power gyro had a mass of $2.0 \mathrm{~kg}$ with $200 \mathrm{~mm}$ external diameter. Its main moments of inertia were $[0.01 ; 0.005 ; 0.005] \mathrm{kg} \cdot \mathrm{m}^{2}$.

The dynamic characteristics of the power gyro are given in Table 2 .

Table 2. The power gyro dynamic characteristics

\begin{tabular}{lccc}
\hline Characteristic & Value & Units \\
\hline $\begin{array}{c}\text { the flywheel velocity } \\
\text { the flywheel moment of inertia with respect to the } \\
\text { axis of rotation }\end{array}$ & 15000 & $\mathrm{rpm}$ \\
& 0.01 & $\mathrm{~kg} \cdot \mathrm{m}^{2}$
\end{tabular}


the power gyro moment of inertia with respect to the axis of actuation (precession)

0.015

$\mathrm{kg} \cdot \mathrm{m}^{2}$

The estimated height of the CoM with respect to the axis of rotation of the inverted pendulum in a stable position was $0.275 \mathrm{~m}$. The estimated height of the common frame was approximately $0.18 \mathrm{~m}$.

We used RDrive 50 and X2216 KV1400 as the motor of the actuation and the motor of the acceleration respectively.

The mechanical parameters for the MATLAB/Simulink model of the system were chosen to fit with those of the experimental plant.

\subsection{The structure of the controller}

At this stage we considered the following conditions and limitations concerning the experimental plant: the sample frequency of the control (5) was $100 \mathrm{~Hz}$ as that of the external computer of the plant; we introduced sensor delays of approximately $10 \mathrm{~ms}$; $4 \cdot 10^{-4} \mathrm{rad} / \mathrm{s}$ additive noise was allowed for the angular velocity estimation. The identified transmission function for the servo actuator was

$$
G(s)=\frac{3.203 s^{2}+1665 s+2.858 \cdot 10^{5}}{s^{3}+55.72 s^{2}+1.013 \cdot 10^{4} s+2.879 \cdot 10^{5}} .
$$

The $\mathrm{Q}$ and $\mathrm{R}$ control parameters were selected based on estimation of quality for the LQR:

$$
J^{i}=\int_{0}^{\infty}\left(x^{T} Q x+u^{T} R u\right) d t, Q=\left(\begin{array}{cccc}
1 & 0 & 0 & 0 \\
0 & 100 & 0 & 0 \\
0 & 0 & 1 & 0 \\
0 & 0 & 0 & 10
\end{array}\right), R=1 .
$$

The absolute value of the control was restricted by $3 \mathrm{rad} / \mathrm{s}$, the backlash was $0.7^{\circ}$. The friction in the axis of rotation of the inverted pendulum was neglected.

The block diagram of the controller in MATLAB/Simulink is depicted in Fig. 2 


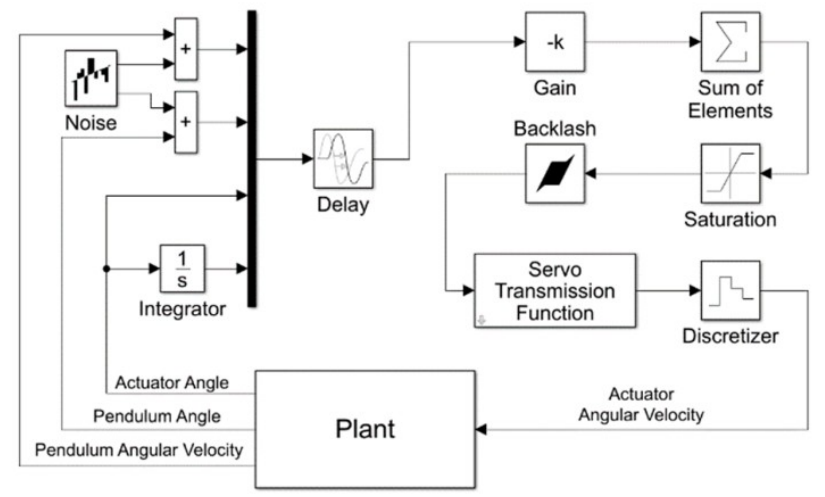

Fig. 2. The block diagram of the control system.

\section{$4 \quad$ Experiment and discussion}

To verify the property of the plant stability in presence of the CoM displacement which was mathematically derived in Subsection 2.3 both the experimental plant and the MATLAB/Simulink model were considered asymptotically stable (or oscillatory stable, due to the explicit non-linearities in the control circuit) in a vicinity of the stationary point $\beta=0, \alpha=0, \dot{\beta}=0, \dot{\alpha}=0$. We then introduced a step perturbation in the form of $\vec{F}=m_{\text {load }} \vec{g}$ applied to the point at the height of $\left(h-\frac{h_{\text {cube }}}{2}\right)$ over the axis of rotation of the inverted pendulum and at the distance of $\frac{h_{\text {cube }}}{2}$ from the axis of symmetry of the inverted pendulum, where $h$ and $h_{\text {cube }}$ were the height of the axis of rotation of the actuator with respect to the axis of rotation of the pendulum and the height of the common frame respectively.

In the case of the experimental plant that was made with the suspension of a load of a similar mass.

The impact started at $t=5 \mathrm{~s}$ after the beginning of the experiment, the mass of the load was $m_{\text {load }}=0.15 \pm 0.01 \mathrm{~kg}$.

The comparison of the experimental graphs for the plant and for the MATLAB/Simulink model is presented in Fig. 3. 

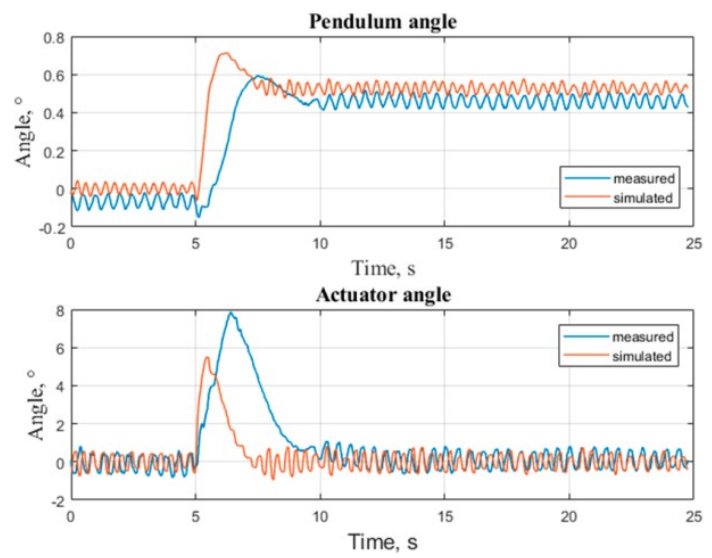

Fig. 3. Experimental graphs for the plant (measured) and for the model (simulated). Top: time dependence of the inverted pendulum angle. Bottom: time dependence of the actuator angle.

From the graphs there is a discrepancy in the characteristics of the transition process for the simulation and the measurements from the experimental plant. The reason for this may be found in some non-linearities lost at the stage of modeling of the system. For example, the misalignment of the axis of actuation and the CoM of the CMG, inaccuracies in calculating the CoM of the construction, and the neglect of friction in the axis of rotation of the inverted pendulum. However, in the graphs shown in Fig. 3, at the end of the transition process the system comes to an asymptotically (oscillating) stable point, which confirms the properties predicted in 2.1 .

\section{Conclusion}

The problem of the article was in the synthesis of the LQR control which should be reliable enough in the case of the CoM-displacement of the CMG-actuated inverted pendulum.

The results of the experiment both on the plant and in the MATLAB/Simulink environment proved the reliability of the proposed LQR, which was able to revise online the equilibrium position of the inverted pendulum.

Further studies will be directed towards more complicated inverted pendulum models with different numbers of CMGs used.

\section{Acknowledgement}

This article was supported by the Ministry of Education and Science of the Russian Federation (project no. 8.2321.2017/4.6). 


\section{References}

1. Ito, S., Nishio, S., Ino, M., Morita, R., Matsushita, K., \& Sasaki, M.: Design and adaptive balance control of a biped robot with fewer actuators for slope walking. Mechatronics, 49, 56-66 (2018).

1. Vorochaeva, L. Y., Yatsun, A. S., Jatsun, S. F.: Controlling a Quasistatic Gait of an Exoskeleton on the Basis of the Expert System. SPIIRAS Proceedings, 3(52), 70-94.

2. Manko, S.V., Shestakov, E.I.: Automatic synthesis of gait scenarios for reconfigurable mechatronic modular robots in the modification of the walking platform. Russian Technological Journal, 6(4), 26-41 (2018).

2. Herra, La P. X. M., Shiriaev, A.S., Freidovich, L.B., Mettin, U., Gusev, S.V.: Stable Walking Gaits for a Three-Link Planar Biped Robot With One Actuator. IEEE Transactions on Robotics 29 (3), 589-601 (2013).

3. Aranovskiy, S., Biryuk, A., Nikulchev, E., Ryadchikov, I., Sokolov, D.: Stabilization of the inverse pendulum with regard to the error in the position sensors. Journal of Computer and Systems Sciences International, 58(2) (2019).

4. Amengonu, Y.H., Kakad, Y.P., Isenberg, D.R.: The Control Moment Gyroscope Inverted Pendulum. In: Swiątek J., Grzech A., Swiątek P., Tomczak J. (eds) Advances in Systems Science. Advances in Intelligent Systems and Computing, vol 240. Springer, Cham (2014).

3. Chu, T.-D., Chen, C.-K.: Design and Implementation of Model Predictive Control for a Gyroscopic Inverted Pendulum. Appl. Sci. 2017, 7, 1272 (2017)

5. Lee, S., Jung, S.: Design of a Fuzzy Compensator for Balancing Control of a One-wheel Robot. International Journal of Fuzzy Logic and Intelligent Systems, 16(3), 188-196 (2016).

6. Aranovskiy, S., Ortega, R., Romero, J. G., Sokolov, D.: A globally exponentially stable speed observer for a class of mechanical systems: experimental and simulation comparison with high-gain and sliding mode designs. International Journal of Control, 1-14 (2017). (doi.org/10.1080/00207179.2017.1404130)

4. Lee, E.B., Markus, L.: Foundations of Optimal Control Theory. Wiley, New York (1967)

7. Anderson, B.D.O., Moore, J.B.: Optimal Control: Linear Quadratic Methods. PrenticeHall, Upper Saddle River, United States of America (1989) 